\title{
Temperature Dependence of the EPR Spectra of the Nanocrystalline TiN and TiC Dispersed in a Carbon Matrix
}

\author{
N. Guskos ${ }^{a, b}$, T. Bodziony ${ }^{a}$, A. Biedunkiewicz ${ }^{c}$ \\ AND K. AIDINIS ${ }^{d}$ \\ ${ }^{a}$ Institute of Physics, Szczecin University of Technology \\ al. Piastów 17, 70-310 Szczecin, Poland \\ ${ }^{b}$ Solid State Section, Department of Physics, University of Athens \\ Panepistimiopolis, 15 784, Greece \\ ${ }^{c}$ Institute of Material Engineering, Szczecin University of Technology \\ al. Piastów 17, 70-310 Szczecin, Poland \\ ${ }^{d}$ Applied Physics Section, Department of Physics, University of Athens \\ Panepistimiopolis, 15 784, Greece
}

Two powder samples: nanocrystalline titanium carbide (TiC) and titanium nitride (TiN) dispersed in a carbon matrix were synthesized by a nonhydrolytic sol-gel process. Both samples were characterized by the X-ray diffraction and transmission electron microscopy. The transmission electron microscopy examination of the $\mathrm{TiC}$ and $\mathrm{TiN}$ nanoparticles showed that their average crystalline size was about $20 \mathrm{~nm}$. The temperature dependence of the EPR spectra for both samples was measured in $10 \mathrm{~K}$ to $200 \mathrm{~K}$ temperature range. A similar very narrow (about $0.2 \mathrm{mT}$ ) EPR line centered at $g \approx 2$ (at room temperature) was recorded in both samples. The EPR line observed in both samples is arising from electron conductivity centers dispersed in the carbon matrix and it was fitted by Dysonian line shape. The temperature dependence of the EPR spectrum showed different behavior of these two samples. It is suggested that in the sample $\mathrm{TiC} / \mathrm{C}$ multiwall carbon nanotubes are formed while in the sample TiN/C the graphite structure dominates.

PACS numbers: 87.64.Hd, 78.67.Bf, 61.46.+w

\section{Introduction}

Titanium carbide $\left(\mathrm{TiC}_{x}\right)$ and titanium nitride $\left(\mathrm{TiN}_{x}\right)$ are very important technological materials because as refractory materials they have gained much at- 
tention due to their extraordinary hardness (wear resistance and stability at very high temperature) and additionally they have a high electrical conductivity [1]. Titanium compounds, especially $\mathrm{TiC}_{x}$ or combination are used in cutting tools and high-temperature alloys for aerospace engineering applications. These compounds are the most intensively studied, both experimentally and theoretically concerning their physical properties, especially the electronic structure, see e.g. $[2,3]$. A few works have appeared devoted to the study of electronic properties by using EPR method [4,5]. The extreme broadness of EPR spectra were observed reflecting the high electrical conductivity of the samples. This electronic behavior is not dissimilar to that of bulk metals, in which the electrons exhibit fairly short spin-lattice relaxation times which give rise to excessively broad lines and render the observation of EPR resonances inaccessible to ordinary spectrometers [4]. Recently, nanocrystalline $\mathrm{TiC} / \mathrm{C}$ and $\mathrm{TiN} / \mathrm{C}$ composites were synthesized by the nonhydrolytic sol-gel process that could be an attractive starting point for low-temperature Ti-C coating technologies [6].

The aim of this report is to present the temperature dependence of the EPR spectra for two titanium compounds dispersed in the carbon matrix $\left(\mathrm{TiC}_{x} / \mathrm{C}\right.$ and $\operatorname{TiN}_{x} / \mathrm{C}$ ) synthesized by a new method, the nonhydrolytic sol-gel process.

\section{Experimental}

The organotitanium gel precursor preparation was described in detail in previous paper $[7,8]$. The gel samples were heated in a furnace in insert and/or reactive atmospheres depending on the desired products. The heating was carried out in gas atmospheres which contained argon and/or nitrogen, hydrogen and ammonia, under the atmospheric pressure in the temperature range from $293 \mathrm{~K}$ to $1273 \mathrm{~K}$. The reactions of the $\mathrm{TiC}$ and $\mathrm{TiN}$ phases proceeded at temperatures below $800 \mathrm{~K}$ during these processes. The parameters of manufacturing of $\mathrm{TiC}$ and TiN with or without carbon as powders are subject to the patenting procedure.

EPR measurements were performed on powder samples sealed in quartz tubes using a Bruker E $500 \mathrm{X}$-band spectrometer $(\nu=9.48 \mathrm{GHz})$ with $100 \mathrm{kHz}$ field modulation and an Oxford flow cryostat for temperature dependent measurements $(4-300 \mathrm{~K})$. The field was scaled with a NMR gaussmeter, while the $g$ factor and EPR intensity were measured with respect to a standard calibrating sample.

\section{Results and discussion}

Figure 1 shows the transmission micrographs of the $\mathrm{TiC} / \mathrm{C}$ sample. Tubes could be seen with diameter of about $50 \mathrm{~nm}$ and length many times greater than diameter. These tubes are too big to be considered as nanotubes but could contain carbon nanotubes inside.

In Fig. 2 and Fig. 3 there are presented the representative EPR spectra of the $\mathrm{TiC}$ and $\mathrm{TiN}$ samples at various temperatures, respectively. The EPR 


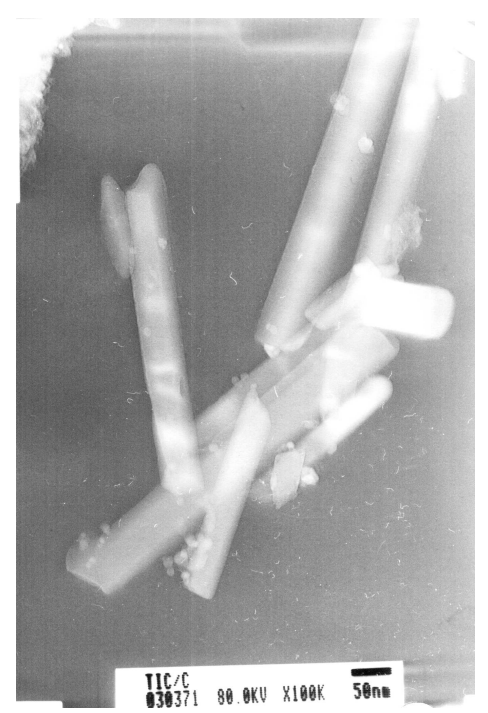

Fig. 1. Transmission electron micrograph (TEM) of the TiC/C sample.
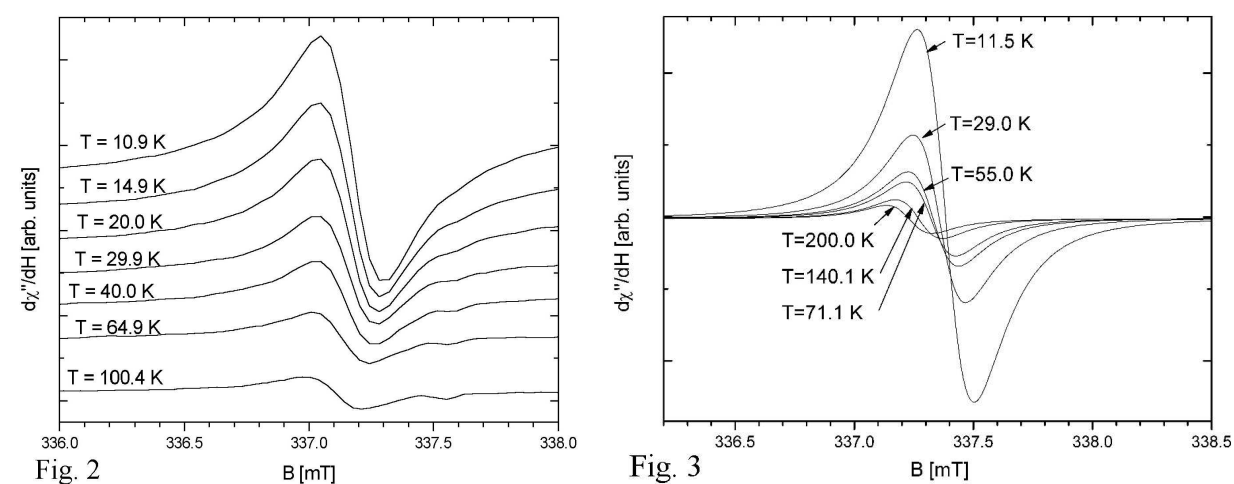

Fig. 2. The EPR spectra temperature dependence for $\mathrm{TiC} / \mathrm{C}$ sample.

Fig. 3. The EPR spectra temperature dependence for TiN/C sample.

line could be fitted very well by using Dysonian line for the sample $\mathrm{TiC} / \mathrm{C}$ and Lorentzian line for the sample TiN/C. It is suggested that the electron conductivity in the sample $\mathrm{TiC} / \mathrm{C}$ is more intense. At temperature $T=298 \mathrm{~K}$, the values of the average $g$ factor and peak-to-peak line width are the following: $g_{\mathrm{av}}=2.0030(5), \Delta H_{\mathrm{pp}}=0.240(2) \mathrm{mT}$ for sample $\mathrm{TiC} / \mathrm{C}$ and $g_{\mathrm{av}}=2.0029(5)$ with $\Delta H_{\mathrm{pp}}=0.185(2) \mathrm{mT}$ for sample $\mathrm{TiN} / \mathrm{C}$ whose $g$ values are very close to the free electron $g_{\mathrm{e}}=2.0023$. Figures 4 and 5 present the temperature dependence of the intensity, peak-to-peak line width, and resonance field for both samples. The temperature behavior of all these parameters is strongly different for these two samples and this suggested that for this fact physical processes could be responsible that make the mechanical properties of $\mathrm{TiC} / \mathrm{C}$ more useful for the applica- 


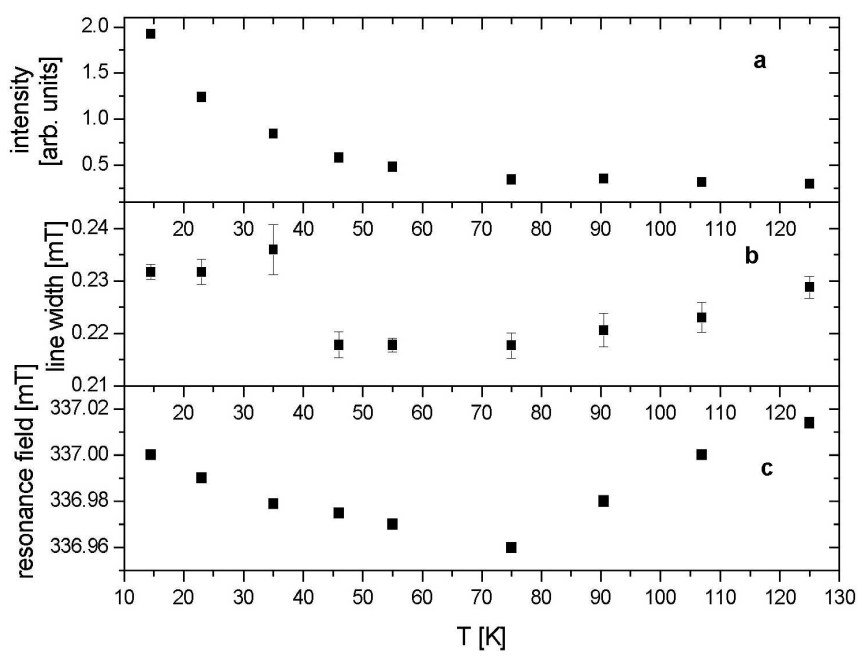

Fig. 4. The temperature dependence of the EPR parameters for the $\mathrm{TiC} / \mathrm{C}$ compound: (a) the intensity, (b) line width, and (c) the resonance field.

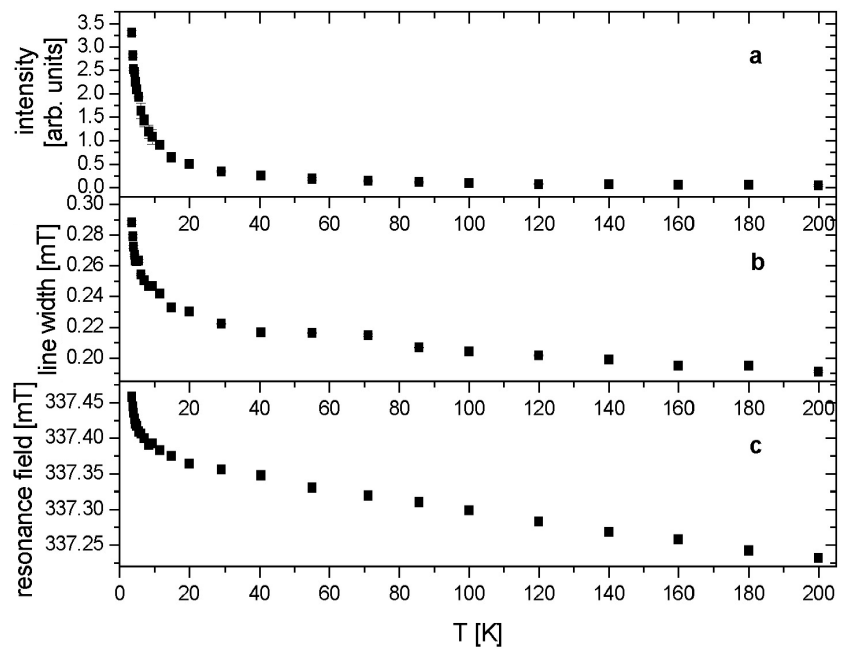

Fig. 5. The temperature dependence of the EPR parameters for the TiN/C compound: (a) the intensity, (b) line width, and (c) the resonance field.

tions. Titanium carbide and titanium nitride compounds have shown the extreme broadness of the EPR spectra due to the superposition of different paramagnetic centers $[4,5]$. It is suggested that the observed EPR line could arise from carbon. The conduction-electron spin resonance (CESR) of graphite and multiwall carbon nanotubes has shown similar EPR spectra [8-12]. The temperature dependence of the $g$ factor could be well described in terms of the band model of quasi-two-dimensional graphite by conduction carriers and additional effect of the localized 
centers $[12,13]$. Thermal annealing effect of carbon did not affect the intensity of CESR spectra but the temperature dependence of $g$ parameter could change significantly after annealing processes [14]. The temperature dependence of the intensity of CESR spectra of $\mathrm{TiC} / \mathrm{C}$ and $\mathrm{TiN} / \mathrm{C}$ compounds up to temperature $60 \mathrm{~K}$ is not changed and it is suggested that the Pauli processes corresponding to the electron conduction are dominating in both samples at higher temperatures (Fig. 4a and Fig. 5a). The peak-to-peak line width and the resonance field behave significantly different for various samples. The peak-to-peak line width has tendency to decrease with decreasing temperature for the sample $\mathrm{TiC} / \mathrm{C}$ up to $60 \mathrm{~K}$ (Fig. 4b) while this dependence is opposite for the sample TiN/N where at lower temperatures strong increase in the line width is observed (Fig. 5b). In both samples near $60 \mathrm{~K}$ an anomaly in the line width is recorded. For the sample $\mathrm{TiC} / \mathrm{C}$ the influence of conducting electrons is more important than for the sample of $\mathrm{TiN} / \mathrm{N}$ as the fitting procedure has shown where the Dyson function and the Lorentz function were used. The temperature dependence of the resonance field has a similar character as the line width (Fig. 4c and Fig. 5c).

If it is supposed that the obtained $g$ parameter for both samples is the result of the motional averaging by conduction carriers, two principal $g$ factors (parallel $-g_{\|}$and perpendicular $\left.-g_{\perp}\right)$ are given by equation $g_{\mathrm{av}}=\left(2 g_{\perp}+g_{\|}\right) / 3$. If $g_{\mathrm{e}}=2.0023$, then experimental $g_{\mathrm{av}}=\left(2 g_{\mathrm{e}}+g_{\|}\right) / 3$ and $\Delta g=g_{\|}-g_{\mathrm{e}}=$ $3\left(g_{\mathrm{av}}-g_{\mathrm{e}}\right)$. According to the $2 \mathrm{D}$ graphite band model the $\Delta g$ could be described by the following relation $[13,14]$ :

$$
\Delta g \approx \frac{3.2 \times 10^{8} \lambda_{\mathrm{eff}} \gamma_{0}^{2}}{(\mathrm{~T}+\delta)^{2}[2+\exp (\eta)+\exp (-\eta)] \ln [2+\exp (\eta)+\exp (-\eta)]},
$$

where $\lambda_{\text {eff }} \approx 2.4 \times 10^{-5} \mathrm{eV}$ is the effective spin-orbit energy interaction, $\gamma_{0} \approx 3 \mathrm{eV}$ is the $2 \mathrm{D}$ band parameter, $\eta=\frac{E_{\mathrm{F}}}{k_{\mathrm{B}}(T+\delta)}$ is the reduced chemical potential determined from the conduction of charge neutrality, $T$ is the lattice temperature and $\delta$ is the additional parameter: $\delta=95(5) \mathrm{K}[12,13]$.

A satisfactory agreement was obtained for the sample $\mathrm{TiC} / \mathrm{C}$ by using relation (1). In the case of TiN/C a good fitting could be achieved if we assume a certain fraction of unpaired electrons localized at the layer defects and the strong exchange interaction between the conduction electrons and localized centers [13]. Spin dynamic fluctuations of the phase transition (metalsuperconductor $)$ is dominating at low temperature $\left(T_{\mathrm{c}}=5.7 \mathrm{~K}\right.$ for TiC compound) and it could have influence on all EPR parameters. As it is seen in the case of $\mathrm{TiC} / \mathrm{C}$, the temperature dependence of EPR parameters behave similar as for the multiwall carbon nanotubes.

\section{Conclusions}

Using new method of preparation two samples: $\mathrm{TiC} / \mathrm{C}$ and $\mathrm{TiN} / \mathrm{N}$ were synthesized and the temperature dependence of their EPR spectra was studied. 
The EPR signal is arising from the conductive carriers in the carbon. Using the same annealing conditions for both samples strongly different temperature behavior of the EPR spectra was observed. It is possible that in the case of $\mathrm{TiC} / \mathrm{C}$ sample the multiwall nanotubes state is formed.

\section{Acknowledgments}

This work has been supported partially by grant No. PBZ-KBN 095/T08/ 2003 of the State Committee for Scientific Research (Poland) 2003-2006.

\section{References}

[1] L.E. Toth, Transition Metal Carbide and Nitrides, Academic Press, New York 1971.

[2] J. Izquierdo, A. Vega, S. Bouarab, M.A. Khan, Phys. Rev. B 58, 3507 (1998).

[3] C. Acha, M. Monteverde, M. Nunez-Requeiro, A. Kuhn, M.A.A. Franco, Eur. Phys. J. B 34, 421 (2003).

[4] A.M. Hassib, A.A.S. Musmus, M.A.A. Issa, Phys. Status Solidi A 89, 147 (1985).

[5] K.J.D. MacKenzie, R.H. Meinhold, D.G. McGavin, J.A. Ripmeester, I. Moudrakovski, Solid State Nuclear Magn. Res. 4, 193 (1995).

[6] A. Biedunkiewicz, Mater. Sci. 21, 445 (2003).

[7] A. Biedunkiewicz, Mater. Sci. 21, 70 (2003).

[8] N. Guskos, A. Biedunkiewicz, J. Typek, S. Patapis, M. Maryniak, K.A. Karkas, Rev. Adv. Mater. Sci. 8, 49 (2004).

[9] G. Wagoner, Phys. Rev. 118, 647 (1960).

[10] K. Matsubara, T. Tsuzuku, K. Sugihara, Phys. Rev. B 44, 11845 (1991).

[11] F. Beuneu, C. l'Huillier, J.P. Salvetat, J.M. Bonard, L. Forro, Phys. Rev. B 59, 5945 (1999).

[12] V. Likodimos, S. Glenis, N. Guskos, C.L. Lin, Phys. Rev. B 68, 045417 (2003).

[13] A.S. Kotosov, D.V. Shilo, Carbon 11, 1649 (1998).

[14] M. Kosaka, T.W. Ebbesen, H. Hiura, K. Tanigaki, Chem. Phys. Lett. 233, 47 (1995). 